\title{
Modeling the time-dependent characteristics of perovskite solar cells
}

\author{
Iman Moeini ${ }^{\mathrm{a}}$, Mohammad Ahmadpour ${ }^{\mathrm{a}}$, Amir Mosavi ${ }^{\mathrm{b}, \mathrm{c}}$, Naif Alharbi ${ }^{\mathrm{e}}$, Nima E. Gorji ${ }^{\mathrm{d}}$ \\ ${ }^{\text {a }}$ Department of Mechanical Engineering, Sharif University of Technology, Tehran, Iran \\ ${ }^{\mathrm{b}}$ Institute of Structural Mechanics, Bauhaus University Weimar, Weimar, Germany \\ ${ }^{\mathrm{c}}$ Institute of Automation, Kando Kalman Faculty of Electrical Engineering, Obuda University, Budapest, Hungary \\ ${ }^{\mathrm{d}}$ Optoelectronics Research Group, Faculty of Electrical and Electronics Engineering, Ton Duc Thang University, Ho Chi Minh City, Viet Nam \\ ${ }^{\mathrm{e}}$ School of Industrial Engineering, Umm Al-Qura University, Saudi Arabia
}

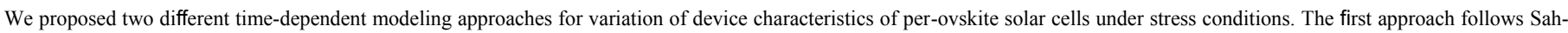

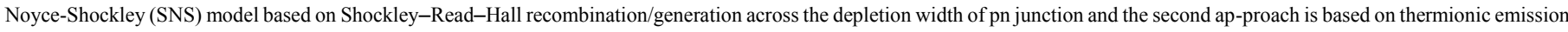

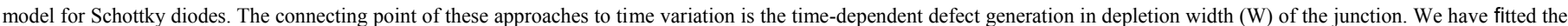

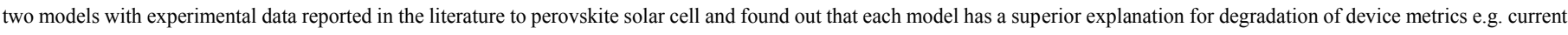
density and efficiency by time under stress conditions. Nevertheless, the Sah-Noyce-Shockley model is more reliable than thermionic emission at least for solar cells.

Keywords:Modeling, Solar cells, Perovskite, Defect generation, Time-dependent

\section{Introduction}

Time dependent models have been rarely developed for curren-t-voltage (JV) characteristics of optoelectronic devices. Time-depen-dent models have much more realistic approaches to device function and provide the observation possibility to determine the degradation/ recovery behavior of a device operating under stress conditions such as long term reverse biasing (e.g. in solar cell, sensors, and photo-detectors) (Alsari et al., 2018; Turturici et al., 2014). The currently available models are presented mainly in static mode which ignores materials and structural changes in the device such as defect generation and intermix of the adjacent layers or in-diffusion of the metallic con-tacts towards the junction. These are the detrimental process that happen by time and cause degradation in device performance. A com-prehensive model must be able to trace the device characteristics by time. We have previously developed several time-dependent theories to model the characteristics of solar cells under stress conditions (Darvishzadeh et al., 2017a; Darvishzadeh et al., 2017b). There are few other publications in the literature which propose time-dependent models for current conduction mechanisms in various devices (Turturici et al., 2017). Turturici et al. have proposed a time dependent modeling for the forward current and reverse biased currents of a photodetector based on p-CdTe (Turturici et al., 2014; Turturici et al., 2017). We will partially use their modeling approach in this paper to develop from a static JV analysis to a time dependent JV curves or at least a current vs. time approach. In their modeling, Turturici et al. have assumed that the defect generation follows an exponential trend by time in the p-type layer and negatively impacts on carrier collection at reverse biases. Although this modeling approach is partly able to explain the current density variation by time, the direct role of electric field at the metal/p-type junction is not clear. A rather parametric model is required to understand how the electron, hole, acceptor, donor defects are involved in the carrier collection under the electricfield in the depletion width of a device. We have previously applied a strong modeling approach to $\mathrm{CdS} / \mathrm{CdTe}$ solar cells which devices the carrier collection to drift and diffusion currents in within and outside of depletion width, respectively (Darvishzadeh et al., 2017a; Darvishzadeh et al., 2017b). Here, we propose the model in time-dependent form for pn junction and photo-detectors based on graphene. We use graphene based devices it has attracted the attention of many researchers not only for solar cell ap-plication but also for sensors, photodetectors, LEDs, etc. over 100 pa-pers have been published last year on graphene application in per-ovskite solar cells (Son et al., 2017; Singh et al., 2018). The recent review on these hybrid devices shows a power conversion efficiency between $10 \%$ and $15 \%$ for graphene and inorganic semiconductorbased hybrid heterojunction solar cells, and $15.6 \%$ for graphene-con-taining perovskite cells. Graphene or carbon nanolayers will act as a supressing layer for shunting process. Bi et al. have designed a 
nanostructured carbon layer to impede the diffusion of ions into per-ovskite layer which have significantly suppressed the degradation process (Bi et al., 2017).

We will develop two time-dependent approaches to model the in-stability of current-voltage characteristics of perovskite solar cells (with graphene contact) under stress conditions of elevated tempera-ture, long term bias or prolonged irradiation. We will fit the model with experimental data reported in literature and will show that our simple but strong modeling can explain the defect generation impact on device characteristics. The modeling is based on a fundamentally different approach than the other theories like transient current and will simply start from Shockley-Read-Hall recombination or Thermionic emission theories.

\section{Modeling approach}

We present two different modeling approach based on SNS theory for the generation/recombination within depletion width and ther-mionic Schottky emission for a fully depleted cell. This theory was well developed by Kosyachenko's group (Kosyachenko et al., 2009; Kosyachenko et al., 2016) and also by our group recently (Aldosari et al., 2016). Both theories have the electric field or depletion width in their formulation which provides us a way to connect them to time and the change in defect density by time. The models provide time-depen-dent current density $\left(\mathrm{J}_{S c}\right)$ and current-voltage characteristics which could lead us to calculate the efficiency variation by time $(\eta(t))$ through solar cell's principal theory (Kosyachenko, 2011).

\subsection{Time-dependent Sah-Noyce-Shockley theory}

The current-voltage characteristics of a pn junction is best described by modeling approach developed by Kosyachenko et al. (2009). The model is based on known Shockley-Read-Hall (SRH) recombination described by Sah-Noyce-Shockley (SNS) theory for the depletion width or as so called space charge region of a pn junction (Kosyachenko et al., 2016),

$$
U(x, V)=\frac{n(x, V) p(x, V)-n_{i}^{2}}{\tau_{p o}\left[n(x, V)+n_{1}\right]+\tau_{n o}\left[p(x, V)+p_{1}\right]}
$$

in the p-type layer,

$n(x, V)=N_{c} \exp -\quad \frac{E_{g}-\mu-\varphi(x, V)-q V}{k T}$,

$p(x, V)=N_{v} \exp -\frac{\mu+\varphi(x, V)}{k T}$.

where $\mu$ is the distance between Fermi level and valence band (conventionally given as: $E_{f}-E_{v}$ ) as shown in Fig. 1. The thermionic emission is not introduced here because the barrier for carrier transport, $\Phi=\varphi_{b i}$ $+\quad \mu$, (characteristic of thermionic emission) does not appear in Eqs.

(1)-(3). $n_{1}$ and $p_{1}$ are determined by SRH theory by supposing that a single defect level $\left(E_{t}\right)$ is located in the band gap,

$n_{1}(x, V)=N_{c} \exp -\frac{E_{g}-E_{t}}{k T} \quad$, where $N_{C}=2\left(m_{n} k T / 2 \pi \hbar\right)^{23 / 2}$

$\underset{1}{p(x, V)=N_{v} \exp } \frac{-E_{t}}{k T}, \quad$ where $N_{v}=2\left(m_{p} k T / 2 \pi \hbar^{2}\right)^{3 / 2}$.

By integrating over $U(x, V)$, the generation current is obtained under reverse bias and recombination current is obtained under forward bias over the SCR (from 0 to W) (Kosyachenko et al., 2009),

$J_{g r}=q \int_{0}^{W} U(x, V) d x$.

where $\mathrm{W}$ is the depletion region of the device where the main

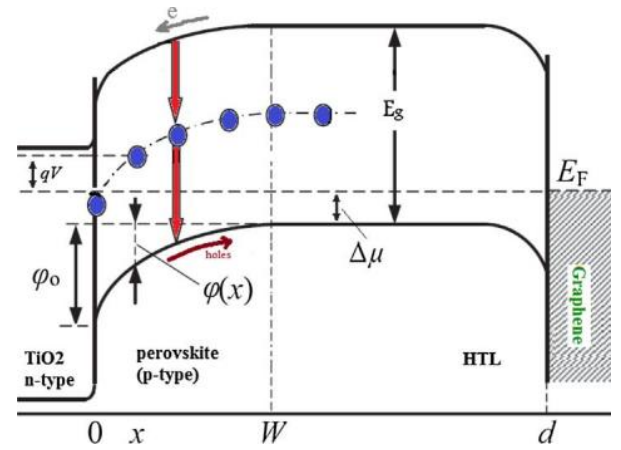

Fig. 1. The energy band diagram of the perovskite solar cell under forward bias. Carrier transport through generation/recombination current, $I_{g r}$, and over-barrier current, $I_{n}$, depletion width, W, band bending $\varphi(x)$, and Fermi levels are indicated (Kosyachenko et al., 2009; Darvishzadeh et al., 2017a). Note that, the back barrier will be neglected for a fully depleted device as $\mathrm{TiO}_{2}$ /perovskite/ HTL layer are thin enough compared to the depletion width $(\mathrm{W}>\mathrm{d})$. Therefore, this diagram changes to a band diagram of a, for example, photodetector with $\mathrm{metal} / \mathrm{semiconductor} / \mathrm{metal}$ structure.

recombination and generation of carriers occurs and the drift current is dominant by a strong electric field. The depletion width is con-ventionally given by Shockley barrier theory,

$W=\sqrt{\frac{2 \in\left(\varphi_{0}-q V\right)}{q^{2}\left(N_{a}-N_{d}\right)}}$,

where $\in$ is the dielectric constant of the p-type layer and $N_{a}$ and $N_{d}$ are acceptor and donor density in depletion region. Note that the term under the root allows modeling both reverse and forward bias ranges only by changing the $+\operatorname{sign}$ to - in $\varphi_{0}-q V$. The static nature of the modeling for current-voltage characteristics comes from this point where $\mathrm{W}$ is taken constant. However, the generation/recombination mechanism is not a static process but a dynamic one which can slightly or significantly change under prolonged irradiation, elevated temperature and ever moisture ingression to the junction (Chen et al., 2016). Therefore, a time-dependent process might be introduced for a real-time analysis of device under operation or at certain times of operation under real conditions. A time-dependent depletion width was introduced by Turturici et. al. for photo-detectors with $\mathrm{Al} / \mathrm{p}-\mathrm{CdTe} / \mathrm{Pt}$ structure (Turturici et al., 2014). They proposed that the depletion width becomes a time-dependent parameter when the defect density exponentially increases in the region,

$W(t)=\sqrt{\frac{2 \in\left(\varphi_{0}-q V\right)}{q^{2} N_{a 0}\left(1-e_{\tau} \bar{\tau}\right)}}$,

where $\tau$ and $N_{a 0}$ are hole detrapping time and deep trap acceptor density at $\mathrm{t}=0$ before the voltage is applied to the photodetector. The time dependent profile of acceptor trap density is given by $N^{-}(t)=N_{T}\left(1-e \frac{-t}{\tau}\right)$ (Turturici et al., 2014). Although we have previously introduced a different defect changing profile to be in form of quadratic or even linear form elsewhere (Darvishzadeh et al., 2017a). By inserting Eq. (8) into Eq. (6) one can calculate a time-dependent photocurrent density for a pn junction device or for a photodetector with a metal/semiconductor/metal structure if the semiconductor thickness $(\mathrm{L})$ is smaller than the depletion width (L W) at all voltages. Finally, Eq. (6) must rewritten in the form of a time-dependent equation,

$J_{g r}(t, x, V)=q \int_{0}^{W(t)} U(x, V) d x$
Note the above is a time, position and voltage dependent equation which makes the modeling quite close to reality. 


\subsection{Time-dependent thermionic emission theory}

Since the perovskite layer is normally very thin of about $300-500 \mathrm{~nm}$, the device will become fully depleted under forward bias or reverse bias. For a fully depleted device we can assume that it's pin structure has reduced to a Shockley diode following the thermionic emission theory. Therefore, the forward and reverse biased perovskite cell can be partially modeled also by a Schottky barrier formed at the $\mathrm{ITO} /\left(\mathrm{TiO}_{2}\right.$-Perovskite junction. $\mathrm{TiO}_{2}$ layer is quite thin less than a $100 \mathrm{~nm}$. The forward current of such a structure is given by the Richardson equation,

$J_{f}(V)=A^{*} T^{2} e \quad \frac{-q \phi b}{k T} \quad \frac{q V}{e k T} \quad-1$

where $\phi_{b}$ is the barrier height at the junction. $\mathrm{V}=\mathrm{V}_{a}-\mathrm{JR}_{\mathrm{S}}$ with $\mathrm{V}_{a}$ and $\mathrm{R}_{\mathrm{S}}$ being the applied voltage and the series specific resistance, respectively.

The barrier height is often bias dependent. Since the perovskite layer was recognized to show dipole piezoelectric material, we can write that the Schottky barrier lowering happens by both image force lowering and dipole nature of perovskite or $\mathrm{TiO}_{2}$ layers (Turturici et al., 2014),

$$
\phi b(V)=\frac{q E}{4 \pi \in}^{1 / 2}+\alpha E
$$

where $\mathrm{E}$ is the electric field in the depletion region. The average of the E-field within $\mathrm{W}$ is given by,

$\bar{E}(x)=\frac{2\left(\phi_{0}-q V\right)}{W}$

where $\phi_{0} \quad$ is the band bending at the junction at $\mathrm{V}=0$. By replacing the $\mathrm{W}(\mathrm{t})$ into above equation, we get,

$\bar{E}(t)=\sqrt{\frac{2 q N_{a 0}\left(1-\frac{-t}{\tau}\right)}{2 \epsilon}}$.

Therefore,the forward current in Eq. (10) becomes time dependent via electric field in the Schottky barrier lowering term. One can again calculate the time-dependent $\mathrm{J}(\mathrm{V})$ graphs by inserting Eq. (10) into Eq. (18). On the other hand, we can also derive the reverse current density $\left(\mathrm{J}_{R}\right)$ of Schottky diode for the thermionic emission model. The $\mathrm{J}_{R}$ in static mode is given by Turturici et al. (2014),

$J_{R}=A^{*} T \exp -\frac{q \phi_{B 0}}{k_{B} T} \exp \frac{q \phi_{b}}{k T}$

where $\mathrm{A}^{*}$ is the effective Richardson constant, $\phi$

is the Schottky barrier height, and $\phi_{b}$ is the Schottky barrier lowering. By inserting Eq. (13) into Eq. (11) and inserting then result into Eq. (14), we get a timedependent reverse current for a thermionic emission (Turturici et al., 2014),

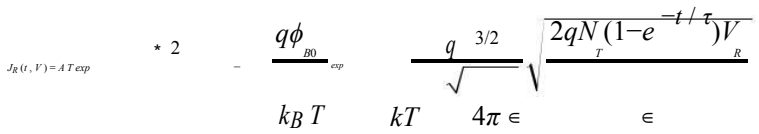

The above equation is valid for transient current-voltage measure-ments where recording a $\mathrm{J}(\mathrm{V})$ graph doesn't take longer than defect ionization process $(\tau)$.

\subsection{Efficiency variation by time}

Now, we can extend our time-dependent model to calculate the variation of the cell efficiency by time. This is straight forward by simply following our previously developed formulation in Ref. Darvishzadeh et al. (2017a) and Darvishzadeh et al. (2017b) where the over-barrier current at the pn junction of a solar cells is given by,

$\begin{array}{cl}J_{n}=q{\frac{n L_{n}}{\tau_{n}}}_{\text {epp }} & \underline{q V} \\ & k T\end{array}$
The total dark current of a solar cell is the sum of above current components,

$J_{d}(t, V)=J_{g r}(t, V)+J_{n}(V)$.

Therefore, $\mathrm{J}(\mathrm{V})$ characteristics of a cell under illumination is given by

$J(t, V)=J_{d}(t, V)-J_{p h}$

where $J_{p h}$ can be taken in the range of $23-25 \mathrm{~mA} / \mathrm{cm}^{2}$ similar to experimentally measured values. Every $\mathrm{J}(\mathrm{V})$ curve of a solar cell under any operation condition or at every certain time results in four basic parameters e.g. $J_{s c}, V_{o c}$, and FF. The latter parameters are time-dependent according to the proposed model. Therefore, one can calculate the efficiency of the cell at a certain time by

$\eta(t)=\frac{J_{S C}(t) V_{o c}(t) F F(t)}{r_{i n}}$,

where $P_{\text {in }} \approx 100 \mathrm{~mW} / \mathrm{cm}^{2}$ is incident power from the sun on the cell. In Eq. (17) one should replace $\mathrm{J}_{g r}$ with $\mathrm{J}_{f}(\mathrm{~V})$ or with $J_{R}(\mathrm{t}, \mathrm{V})$ when fitting the data with the time-dependent thermionic emission model.

\section{Modeling results and discussion}

The stability of a solar cell is a crucial issue for its commercial application. However, the long-time stability of organic-inorganic PSC is still unsatisfactory.

We will now apply the theory to a perovskite solar cell with graphene contact. Fig. 2 shows the JV curves calculated at different times using Eq. (19) with the current density inserted from the time-dependent SNS theory (Eq. (9). The JV curves calculated at $t=0$ compared to $\mathrm{JV}$ curves calculated at $\mathrm{t}=10 \mathrm{~s}$ and $20 \mathrm{~s}$ which shows a clear difference between the device metrics such as $\mathrm{J}_{s c}$ and $\mathrm{V} o c$ values. The cell was assumed to be under sever stressing conditions which eventually cause extreme defect generation rate with exponential increment as been used in Eq. (8). It is observed that the curves show different $J_{s c}$ and $V_{o c}$ values at different times but no change in FF is obtained. This mostly arise from missing $R_{S}$ term in the modeling approach since it is known that the change in FF of a solar cells is related to series resistance of the cell. We expect that the FF of the cell must reduce by time as the defect generation will increase the series resistance. However, the square shape of the curves show no change by time which is a deficiency of the model and can be corrected by adding the $\mathrm{V} / R_{S}$ term in the modeling (Domanski et al., 2015). In the inset of the figure, we have also shown that the device has a graphene back contact which can eventually

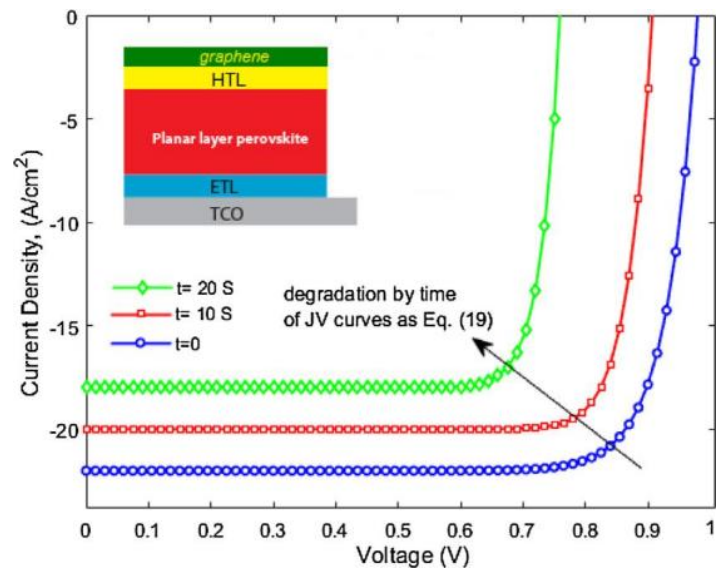

Fig. 2. The modeling results of current-density characteristics of a perovskite based solar cell with reduced-graphene-oxide back contact modeled for three different transient times at $\mathrm{t}=0,10 \mathrm{~s}$ and $20 \mathrm{~s}$. The model is showing a lower $J_{S c}$ and $V_{O c}$ for higher times in line with experimental data presented in Singha and Singh Nalwa (2015) 


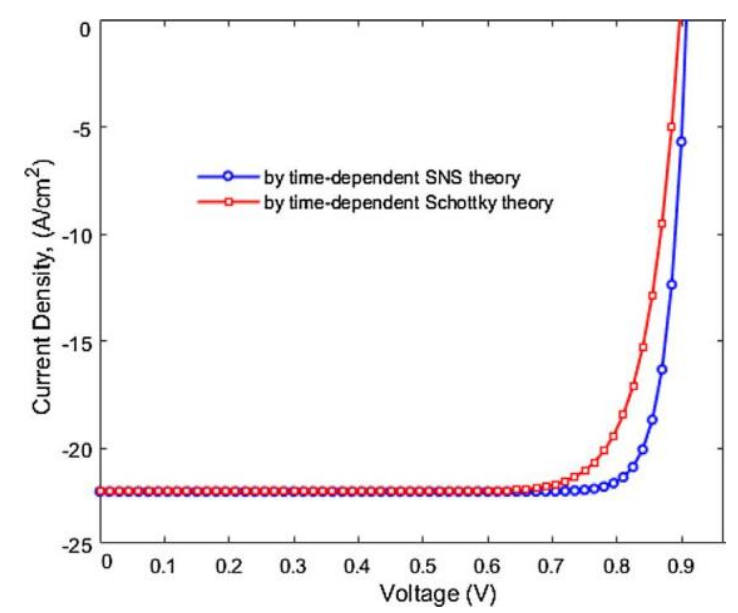

Fig. 3. Current-voltage characteristics of perovskite based solar cell fitted by to different approach modeled by SNS theory and Thermionic emission theory. By looking at FF values (closer fit to a square shape of the curves), a better results are obtained by SNS theory which takes into account the recombination and generation rate in depletion width of the cell.

reduce the series resistance from the HTL/graphene junction. Alsari et al. have shown that efficiency drop of perovskte cells under stress condition is mainly due to a reduction in $J_{S C}$, however, the $V_{O C}$ also re-duces in such devices (Alsari et al., 2018).

Fig. 3 shows the comparison of two models where the time-depen-dent current density calculated by both models are compared. Clearly, we see a better FF for the curve calculated out of time-dependent cur-rent density of SNS theory. This might be due to the fact that the SNS theory is has more number of fitting parameters than the Thermionic emission theory which is basically for a semiconductor/metal structure rather than a pin perovskite diode. It has been well demonstrated that the degradation of the charge extraction layers (or ETL/HTL interfaces) under stress, plays a significant role in disturbing the charge extraction mechanism.

Fig. 4 shows the normalized current-density vs. transient time of the cell under stress. Both models were plotted on to fit with the experi-mental data reported in literature. We fitted the models with data re-ported in Ref. Singha and Singh Nalwa (2015) but many other refer-ences are showing the same trends (Islam et al., 2017). The comparison of two models show that the SNS theory fits better with data in longer times while the thermionic emission fits in shorter times. We also plotted the variation of defect generation by time in the depletion width

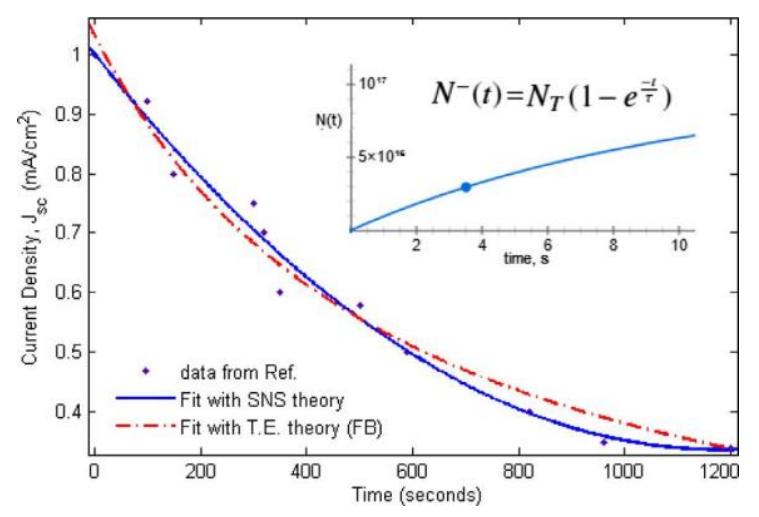

Fig. 4. Normalized current density vs. time: Fitting the experimental data re-ported in Ref. Singha and Singh Nalwa (2015) with both SNS theory and Thermionic Emission (TE) theory for forward bias (FB) region. A better fit is obtained with SNS theory which is based on generation/recombination theory. The inset shows (in scale) the variation of depletion width by time due to defect increment. of the perovskite cell. Turturici et al. have shown that the degradations of performance over time under bias is a major drawback of Schottky contact in CdTe photodetectors (Turturici et al., 2017). This phenom-enon is known as polarization induced due to charge accumulation in depletion width. Hou et al. have measured the transient current of perovskite cells under stress of illumination (Hou et al., 2017). They reported that the reduced carrier recombination in perovskite cells under illumination by time can efficiently improve carrier separation and extraction due to defect annihilation when the light is switched off. These mechanisms have been proven to be time-dependent Luo et al. have used graphene oxide as the back contact or modifying dopant to hole transport layer (HTL) which is PEDOT:PSS in perovskite cells and have shown that reducing graphene oxide (GO) increases the photo-absorption and diminishes the charge recombination across grain boundaries and at the perovskite/HTL interface (Luo et al., 2017). The charge transporting and charge collection probability for an effective voltage can directly obtained from $\mathrm{J}_{p h} / \mathrm{J}_{\text {sat }}$ ratio. The charge trans-porting and collecting probability of cells under the Jsc condition in-creased from $98.03 \%$ in the control device to $99.32 \%$ in a cell with the GO modification, indicating that modifying GO improves the hole passivation and charge collection abilities of the cells.

Fig. 5 shows the variation of normalized energy conversion effi-ciency versus transient time of the perovskite cells under stress. Both models were reasonably fit with the experimental data reported in lit-erature. The data were extracted from literature (Ref. Singha and Singh Nalwa (2015)). The inset of the figure shows a quantitative decrease of the depletion width by time due to defect generation. We have per-formed a numerical calculation on $\mathrm{W}$ changing by time due to defect increment which has been presented in Ref. Aldosari et al. (2016). Our modeling results are consistent with in-diffusion of defective ions into absorber layer of solar cells. One can assume that these ions modify the electronic properties of the FTO/perovskite interface and can raise in an exponential trend increasing by time. The model can be used to fit with the measured data under reverse bias. In this case the reverse bias theory of by thermionic emission can be used. One can also use the models to model the $\mathrm{V}_{o c}(\mathrm{t})$ or $\mathrm{FF}(\mathrm{t})$ in both forward to reverse bias re-gimes. The change in defect density across the perovskite layer can change it's conductivity and the fill factor finally. The graphene layer in this case, can reduce the defect/ion diffusion into the perovskite layer and reduce the degradation probability.

These theories can also be modified to fit with the current transient measurements on perovskite cells (Chen et al., 2017). The models are also valid to fit with recovery trend in various semiconductor devices (Liao et al., 2017). We also believe that the artificial intelligence

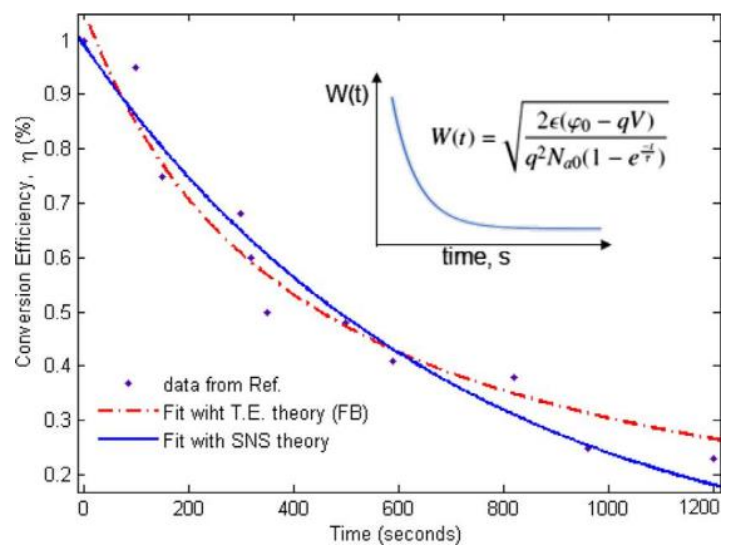

Fig. 5. Normalized energy conversion efficiency vs. time: Fitting the experi-mental data reported in Ref. Singha and Singh Nalwa (2015) with both SNS theory and Thermionic Emission (TE) theory for forward bias (FB) region. A better fit is obtained with SNS theory which is based on generation/re-combination theory. The inset shows (in scale) the defect increment by time via an exponential function. 
technoque can be used to get the engagement of other parameters into account for modeling the time-dependent behaviour of solar cells (Yang and $\mathrm{Yu}, 2017$; Li et al., 2017; Weber, 2018; Wang et al., 2018; Henderson et al., 2018).

\section{Conclusion}

We have developed two different time-dependent modeling for degradation or recovery of the device characteristics of several perovskite solar cells. The two models are primarily based on Sah-Noyce-Shockley theory and conventional Schottky thermionic emission. These are then connected to defect generation in the depletion width by time and thus make the current-voltage characteristics a time-dependent figure. We have correctly assumed that the defect (deep acceptor) density ex-ponentially increases by time under stress conditions and thus changes the depletion width, electric field and current density a not static but a time-dependent parameter. The models were fitted with experimental data reported in literature on current density, JV and efficiency varia-tion by time under stress conditions such as longer term biasing the cells. It is concluded that device physics of solar cells must be con-sidered as a timedependent process instead of static process since the defect generation and annihilation is a dynamic and kinetic process and a transient kinetic model will rather be realistic. The defect generation occurs in most stressing or normal operation conditions and that will change the device modeling non-static. The models used here have been fitted with literature data and we observed that SNS theory or TE theory fit with part of the data and cannot fully explain the entire data of all times. That shows the significant importance of a time-dependent approach in modeling the device physics of solar cells.

\section{References}

Aldosari, M., Grogoriu, L., Sohrabpoor, H., Gorji, N.E., 2016. Modeling of depletion width variation over time in thin film photovoltaics. Modern Phys. Lett. B 29, 1650044.

Alsari, M., Pearson, A.J., Wang, J., Wang, Zh., et al., 2018. Degradation kinetics of in-verted perovskite solar cells. Sci. Rep. 8, 5977.

Bi, E., Chen, H., Xie, F., Wu, Y., Chen, W., Su, Y., Islam, A., 2017. Diffusion engineering of ions and charge carriers for stable efficient perovskite solar cells. Nature Commun. 8, 15330.

Chen, J., Zhou, S., Jin, S., Lia, H., Zhai, T., 2016. Crystal organometal halide perovskites with promising optoelectronic applications. J. Mater. Chem. C 4, 11.

Chen, B., Li, W.G., Rao, H.S., Xu, Y.F., Kuang, D.B., Su, Ch.Y., 2017. Large-grained per-ovskite films via FAMAPb(IBr)3 single crystal precursor for efficient solar cells. Nano Energy 34, 264-270.
Darvishzadeh, P., Redzwan, Gh., Ahmadi, R., Gorji, N.E., 2017a. Modeling the degradation/recovery of short-circuit current density in perovskite and thin film photo-voltaics. Org. Electron. 43, 247-252.

Darvishzadeh, P., Babanezhad, M., Ahmadi, R., Gorji, N.E., 2017b. Modeling the degradation/recovery of open-circuit voltage in perovskite and thin film solar cells. Mater. Des. 114, 339-344.

Domanski, K., Tress, W., Moehl, T., Saliba, M., Nazeeruddin, M.K., Grtzel, M., 2015. Working principles of perovskite photodetectors: analyzing the interplay between photoconductivity and voltage-driven energy-level alignment. Adv. Funct. Mater. 25, 6936-6947.

Henderson, J.-P., Plummer, A., Johnston, N., 2018. An electro-hydrostatic actuator for hybrid active-passive vibration isolation. Int. J. Hydromechatron. 3 (1), 47-71.

Hou, X., Pan, L., Huang, S., Ou-Yang, W., Chen, X., 2017. Enhanced efficiency and stability of perovskite solar cells using porous hierarchical $\mathrm{TiO}_{2}$ nanostructures of scattered distribution as scaffold. Electrochim. Acta 236, 351-358.

Islam, M.B., Yanagid, M., Shirai, Y., Nabetani, Y., Miyano, K., 2017. NiO hole transport layer for perovskite solar cells with improved stability and reproducibility. ACS Omega 2, 2291-2299.

Kosyachenko, L.A., 2011. Solar Cells-Thin-Film Technologies. InTech, Croatia. Kosyachenko,

L.A., Savchuk, A.I., Grushko, E.V., 2009. Dependence of efficiency of thinfilm CdS/CdTe solar cell on parameters of absorber layer and barrier structure. Thin Solid Films 517, 2386-2391.

Kosyachenko, L.A., Lytvynenko, V.Yu., Maslyanchuk, O.L., 2016. Quantitative analysis of optical and recombination losses in $\mathrm{Cu}(\mathrm{In}, \mathrm{Ga}) \mathrm{Se} 2$ thin-film solar cells. Semiconductors 50 (4), 508-516.

Liao, J.-F., Li, W.G., Rao, H.-Sh., Chen, B.-X., Wang, X.-D., Chen, H.-Y., Kuang, D.-B., 2017. Inorganic cesium lead halide $\mathrm{CsPbX} 3$ nanowires for long-term stable solar cells. Sci. China Mater 60 (4), 285-294.

Li, Ch., Zhang, Y., Tu, W., Jun, C., Liang, H., Yu, H., 2017. Soft measurement of wood defects based on LDA feature fusion and compressed sensor images. J. Forestry Res. 28 (6), 1285 1292 .

Luo, H., Lin, X., Hou, X., Pan, L., Huang, S., Chen, X., 2017. Efficient and air-stable planar perovskite solar cells formed on graphene-oxide-modified PEDOT:PSS hole transport layer. Nano-Micro Lett. 9, 39.

Singha, E., Singh Nalwa, H., 2015. Stability of graphene-based heterojunction solar cells. RSC Adv. 5, 73575.

Singh, M., Kumar, G., Prakash, N., Khanna, S.P., 2018. Large bandgap reduced graphene oxide (rGO) based np heterojunction photodetector with improved NIR performance. Semicond. Sci. Technol. 33, 045012 .

Son, S.B., Huang, F., Bae, T.S., Hong, W.K., 2017. Wettability effects of graphene oxide aqueous solution in photodetectors based on graphene oxide/silicon heterojunctions via ultraviolet ozone treatment. J. Alloys Compd. 698, 384-389.

Turturici, A.A., Abbene, L., Gerardi, G., Principato, F., 2014. Electrical characterization of CdTe pixel detectors with Al Schottky anode. Nucl. Instrum. Methods Phys. Res. A 763, 476-482.

Turturici, A.A., Franc, J., Grill, R., Principato, F., 2017. Electric field manipulation in Al/ CdTe/Pt detectors under optical perturbations. Nucl. Instrum. Methods Phys. Res. A 858, 36-43.

Wang, Zh., Xie, Zh., Huang, W., 2018. A pin-moment model of flexoelectric actuators. Int. J. Hydromechatron. 3 (1), 72-90.

Weber, J., 2018. Independent metering systems. Int. J. Hydromechatron. 3 (1), 107-125. Yang,

H., Yu, L., 2017. Feature extraction of wood-hole defects using wavelet-based ultrasonic testing. J. Forestry Res. 28 (2), 395-402.

Darvishzadeh, A., Alharbi, N., Mosavi, A., \& Gorji, N. E. 2018. Modeling the strain impact on refractive index and optical transmission rate. Physica B: Condensed Matter, 543, 14-17.

Moeini, I., Ahmadpour, M., Mosavi, A., Alharbi, N., \& Gorji, N. E. 2018. Modeling the detection efficiency in photodetectors with temperature-dependent mobility and carrier lifetime. Superlattices and Microstructures. 
